\title{
Learning Word Clusters from Data Types
}

\author{
Paolo Allegrini, Simonetta Montemagni, Vito Pirrelli \\ Istituto di Linguistica Computazionale - CNR \\ Via della Faggiola 32 , Pisa, ltaly \\ \{allegrip,simo,vito\}@ilc.pi.cnr.it
}

\begin{abstract}
The paper illustrates a linguistic knowledge acquisition model making use of datia types, infinite memory, and an inforential mechanism for inducing new information from known data. The model is compared with standard stochastic methods applied to data tokens, and tested on a task of lexico-semantic classification.
\end{abstract}

\section{Introduction and Background}

Of late, considerable interest has been raised by the use of local syntactic contexts to automatically induce lexico-semantic classes from parsed corpora (Pcreira and Tishby 1992; Percira et al. 1993; Rooth 1995; Rooth et al. 1999). This family of approaches takes a pair of words (usually a verb plus a nomn), and a syntactic relation bolding between the two in context (usually the object), and calculates its token distribution in a training corpus. These counts define the range of more or less typical syntactic coljocates selected by a verb. The semantic similarity between words is then defined in terms of substitutability in local contexts (sce also Grefenstettc 1994; Lin 1998): two verbs are semantically close if they typically share the same range of collocates; conversely, two nouns are semantically close if they take part in the same type of selection dependencies, i.e. if they are selected by the same verbs, with the same function. From this perspective, a syntiactically asymmetric relation (a dependency) is reinterpreted as a semantic co-selection, where each term of the relation can be defined with respect to the other.

This symmetric similarity metric is often accompanied by the non trivial assumption that the semantic classification of both verbs and nouns be symmetric too. This is enforced by $\operatorname{maximizing} \prod p\left(v_{i}, n_{j}\right)$, with

$$
p\left(v_{i}, n_{j}\right) \simeq \sum_{l=1}^{\omega} p\left(C_{t}\right) p\left(v_{i} \mid C_{l}\right) p\left(n_{j} \mid C_{t}\right)
$$

where $p\left(C_{k}\right)$ is the probability of class $C_{k}$ being found in the training corpus, and $p\left(v_{i} \mid C_{k}\right)$ and $p\left(n_{j} \mid C_{k}\right)$ define the probability that verb $v_{i}$ and nown $n_{j}$ be associated with the semantic dimension (or meaning component) of class $C_{k}$. Intuitively, the joint distribution of functionally ammotated verb-noun pairs is accounted for by assuming that cach pair momber independently correlates with the same semantic dimension, or selection type (Rooth 1995), a conceptual pair defining all pairs in the class: e.g. "scalar motion", "commmicative action" etc.

The approach has the potential of dealing with polysemous words: as the same word can in principle belong to more than one class, there are good reasons to expect that the corresponding selection type positively correlates with onc and only one sense of polysemous words. A further bonus of the approach is that it makes it explicit the perspectivizing factor underlying the discovered similarity of words in a class.

On a less positive side, poorly selective verbs (i.e. verbs which potentially combine with any noun) such as give, find or get tend to stick together in highly probable classes, but appear to stake out rather uninformative semantic dimensions, relating a motley collection of nouns, such as part, way, reason and problem (Rooth 1995), whose only commonality is the property of being freely interchangeable in the context of the above-mentioned verbs.

Another related issue is how many such dimensions are necessary to account, for the entire variety of senses attested in the training corpus. This is an empirical question, but we contend 
an important one, as the usability of the resulting classes heavily depends on it. It is common knowledge that verbs can be exceedingly choosy in the way they select their collocates. Hence, one is allowed to use the class $C_{k}$ to make predictions about the set of collocates of a verb $v_{i}$, only if $P\left(v_{i} \mid C_{k}\right)$ is sufficiently high. Conversely, if $C_{k}$ happens to poorly correlate with any verb, the set of nouns in $C_{k}$ is unlikely to reflect any lexical solection. This compounds with the problem that the meaning of a verb $v_{i}$ can significantly involve more than one semantic dimension: at the prosent stage of research in computational lexical semantics, no scholar has shown what function rolates the moaning components of $v_{i}$ to its selectional behaviour. There is wide room for further research in this area, but truly explorative tools are still necded.

Finally, the described methor is acutely prone to the problem of sparse data. Mlthough $p(C \mid n)$ is rightly expected to converge faster than $p(v \mid n)$, still convergence of $p(C \mid n)$ can bo exceedingly slow with low freguency nomus. It is moot that sieving more and more corpus data is a solution in all casces, as word frequency is highly sensitive to changes in toxt genre, topice and domain (Schintzo and Pedersen 1993).

\section{The approach}

Ilere we illustrate a diflerent apperoach to acquiring lexico semantic: classes from syntiactically local contexts. Jike the family of stochiastic methods of section d, we make use of a similarity metric based on substitutability in (verb,noun,function) triples. We also share tho assumption that lexico-semantic classes are inherently multidimensional, as they hoavily depend on existence of a perspectivizing factor.

Yet, we depart from other assumptions. Classification of verbs and nouns is asymmetric: two nouns are similar if they collocate with as many semantically diverse verbs as possible in as many different syntactic contexts as possible. The converse applies to verbs. In other words, semantic similarity of nouns is not conditional on the similarity of their accompanying verbs, and viceversa. In a sense, classification breaks the symmetry: maximization of the similarity of nouns (verbs) may cause minimization of the sinnilarity of their accompanying verbs (nouns). A class where a maximum of noun similarity correlates with a maximum of verb similarity can be uninformative, as exemplified above by the case of poorly selective verbs.

Secondly, wo assume (following Fodor 1998) that the number of perspectivizing factors governing lexical selection may have the order of magnitude of the lexicon itself. The use of global semantic dimensions may smooth out lexical preferences. This is hardly what wo need to semantically annotate lexical preferences. $\Lambda$ more conservative approach to the problem, inducing local semantic (lasses, can combine alpplicability to real language -processing problems with the further bonus of exploring a relatively uncharted territory.

'Thirdly, $p\left(v_{i}, n_{j}\right)$ appears to be too sensitive to changes in text genre, topic and domain to be expected to converge reliably. We prefer to ground a similarity metric on measuring the correlation among verb-noun types rather than tokens, for two basic reasons: i) verb-noun types are discrete and less prone to random variation in a (parsed) corpus, ii) verb-noun types (an reliably be accuired firom highly informative but hardly redundant knowledge sources such as lexica and encyclopacdias.

Finally, our information unit for measuring word similarity is not a couple of contextsharing pairs (e.g. (set,standard,obj) and (sct,record,obj)) but a quadruple of such contexts, formed by combining two verbs with two 11oums (e.g. (sel,standard,obj), (set,yecord,obj), (miss, standard,ol, $\mathrm{j})$ and (miss, record,ob $\mathrm{j})$ ), such that they enter: an analogical proportion.

\subsection{The analogical proportion}

In the present context, an analogical proportion (hereafter $A P$ ) is a quadruple of functionally annotated pairs resulting from the combination of any two nouns $n_{i}$ and $n_{j}$ with any two verbs $v_{k}$ and $v_{t}$ such as (2) holds:

$$
\begin{array}{cl}
\left(v_{k}, n_{i}, f_{m}\right): & \left(v_{k}, n_{j}, f_{m}\right)= \\
\left(v_{t}, n_{i}, f_{n}\right): & \left(v_{t}, n_{j}, f_{n}\right),
\end{array}
$$

where terms along the two diagonals can swap place in the proportion, and identity of subscript indicates identity of values. 'T'hree aspects of (2) are worth emphasizing in this context. First, it does not require that the same syntactic function hold between all pairs, but only that functions be pairwise identical. Moreover, (2) does not cover all possible syntactic contexts where $n_{i}, n_{j}, v_{k}$ and $v_{t}$ may combinc, but only 
those where verb and function values co-vary.

$$
\begin{aligned}
(\text { set, standard, obj) } & :(\text { set, record }, o b j)= \\
(\text { meet, standard, obj }) & : \quad(\text { meet }, \text { record }, x)
\end{aligned}
$$

We call this constraint the "same-verb-samefunction" principle. As we will see in section 2.3, the principle has important consequences on the sort of similarity induced by (2). Finally, if one uses subscripts as formal constraints on type identity, then any term can be derived from (2) if the values of all other terms are known. For example given the partially instantiated proportion in (3), the last term is filled in unambiguously by substituting $x=f_{n}=o b j$.

$A P$ is an important generalization of the inter-substitutability assumption, as it extends the assumption to cases of functionally heterogencous verb-noun pairs. Intuitively, an $A P$ says that, for two nouns to be taken as systematically similar, one has to be ready to use them interchangeably in at least two different local contexts. This is where the inferential and the classificatory perspectives meet.

\subsection{Mathematical background}

We gave reasons for defining the similarity metric as a function of verb-noun type corrclation rather than verb-noun token correlation. In this section we sketch the mathematical framework underlying this assumption, to show that, for a set of verb-noun pairs with a unique syntactic function, $A P$ is the smallest $C$ that satisfies eq.(1).

Eq.(1) says that $v_{i}$ and $n_{j}$ are conditionally independent given $C$, meaning that their correlation only depends on the probability of their belonging to $C$, as formally restated in eq.(4).

$$
p(n, v \mid C)=p(n \mid C) p(v \mid C)
$$

In passing from token to type frequency, we assume that a projection operator simply assigns a uniform type probability to cach event (pair) with a nonzero token probability in the training corpus. From a learning perspective, this corresponds to the assumption that an infinite memory filters out events alrcady seen during training. The type probability $p_{T}(n, v)$ is defined as in eq.(5), where $N_{p}$ is the number of different pairs attested in the training corpus.

$$
\begin{aligned}
& p_{T}(n, v)=1 / N_{p} \text { if the pair is attested } \\
& p_{T}(n, v)=0 \text { otherwise. }
\end{aligned}
$$

By eq. (4), $p_{T}(n, v \mid C) \neq 0$ if and only if $p_{T}(n \mid C) \neq 0$ and $p_{T}(v \mid C) \neq 0$. This amounts to saying that all verbs in $C$ are freely interchangeable in the context of all nouns in $C$, and viceversa. We will hercafter refer to $C$ as a $s u b$ stitutability island $(S I)$. $A P$ can accordingly be looked at as the minimal $S I$.

The strength of correlation of nouns and verbs in each $S I$ can be measured as a summation over the strength of all $A P$ s where they enter. Formally, one can define a correlation score $\sigma(v, n)$ as the probability of $v$ and $n$ being attested in a pair. This can be derived from our definition of $p_{T}(v, n)$, as shown in eq. (6), by substituting $p_{T}(n, v)=p_{T}(v) p_{T}(n \mid v)$ and $p_{T}(n \mid v)=1 / \omega(v)$, where $\omega(a)$ is the type frequency of a (i.e. number of different attested pairs containing $a)$.

$$
\begin{array}{r}
\frac{1}{N_{p}}=p_{T}(n, v)=p_{T}(n) p_{T}(v) \sigma(n, v)= \\
\frac{\omega(n)}{N_{p}} \frac{\omega(v)}{N_{p}} \sigma(n, v)
\end{array}
$$

Eq.(6), after simplification, yiclds the following

$$
\sigma(n, v) \propto[\omega(n) \omega(v)]^{-1} .
$$

By the same token, the correlation function $\sigma(A P)$ relative to the 4 possible pairs in $A P$ is calculated as

$$
\begin{array}{r}
\sigma(A P)=p_{T}\left(v_{1} \mid n_{1}\right) p_{T}\left(n_{2} \mid v_{1}\right) p_{T}\left(v_{2} \mid n_{2}\right) p_{T}\left(n_{1} \mid v_{2}\right) \\
\propto\left[\omega\left(n_{1}\right) \omega\left(v_{1}\right) \omega\left(n_{2}\right) \omega\left(v_{2}\right)\right]^{-1} .
\end{array}
$$

Eq.(8) captures the intuition that the correlation score between verbs and nouns in $A P$ is an inverse function of their type frequency. Nouns and verbs with high type frequency occur in many different pairs: the less selective they are, the smaller their semantic contribution to $\sigma(A P)$.

Our preference for $\sigma(A P)$ over $\sigma(v, n)$ underlies the definition of correlation score of $S I$ given in eq.(9) (see also section 4).

$$
\sigma(S I) \equiv \sum_{A P \in S I} \sigma(A P) .
$$

\subsection{Breaking the symmetry}

In section 2.2 we assumed, for the sake of simplicity, that verbs and nouns are possibly related through one syntactic function only. In a 
proportion like (2), however, the syntactic function is allowed to valy. Nonetheless each related $S I$ contains nomns which always combine with a given verb with one and the same symtactic: function. Clearly, the same is not true of verbs. Suppose that an $S I$ contains two verbs $v_{k}$ and $v_{t}$ (say drive and pierce) and two nouns $n_{i}$ and $n_{j}$ (say nail and peg) that are respectively object and subject of $v_{k}$ and $v_{t}$. The type of similarity in the resulting nom and verb clusters is of a completely different, nature: in the case of nomus, we accuire distributionally parallel words (c.g. nail and peg); in the case of verlos, wo get distributionally correlated words (say drive and pierce) which are not interchangeable in the same context. Mixing the two types of distributional similarity in the same class makes little sense. Hereafter, we will aim at maximizing the similarity of distributionally parallol nouns. In doing so, we will use functionally heterogeneous contexts as in (2). 'This breaks classification symmetry, and there is no guarantee that semantically coherent verb chusters be returned.

\section{The method}

The section illustraties an application of the principles of section 2 to the task of clustering the set of oljects of a verb on the basis of a repository of functionally inmotated contexts.

\subsection{The knowledge base}

The training evidence is a Knowledge Base $(K B)$ of functionally annotated verb-nom pairs, instantiating a wide range of syntactic relations:

a) verb object, e.g. (causare, problema, ob) 'cause-problem';

b) verb-subject, c.g. (capitare, problema, subj) 'occur-problem';

c) verb-prepositional_complement, e.g. (incappare, problema, in) 'run_into-problem'.

The $K B$ contains 43,000 pair types, automatically extracted from different knowledge sources: dictionaries, both bilingual and monolingual (Montemagni 1995), and a corpus of financial newspapers (Federici et al. 1998). 'The two sources reflect two different modes of lexical usage: dictionaries give typical examples of use of a word, and rumning corpora attest actual usage of words in specific embedding domains. These differences have an impact on the typology of senses which the two sources provide evidence for. General dictionaries testify all possible senses of a given word; typical word collocates accuired from dictionaries tend to cover the entire range of possible senses of a headword. On the other hand, unrestricted textis rellect actual usage and possibly bear witness to senses which are relevant to a specific domain only.

\subsection{The input words}

There is abundant psycholinguistic evidence that semantic similarity between words is emincently context-sensitive (Miller and Charles 1991). Moreover, in many language-processing tasks, word similarity is typically judged relative to an actual context, as in the cases of syntactic disambiguation (both structural and functional), word sense disambiguation, and selection of the contextually appropriate translation equivalent of a word given its neighbouring words. Finally, close examination of real data shows that different word senses select classes of complements according to different dimensions of semantic similarity. This is so pervasive, that it soon becomes impossible to provide an effective account of these dimensions independently of the sense in cuestion.

Evaluation of both accuracy and usability of any automatic classification of words into scmantic clusters camnot but artificially elude the basic question "similar in what respect?". Our choice of inputi words reflects these concerns. We automatically clustered the set of objects of a given verb, as they are atitested in a test corpus. This yiclds local lexico-semantic classes, i.c. conditional on the selected verb head, as opposed to global classes, i.e. built once and for all to account for the collocates of any verb.

Among the practical advantages of local classification we should at least mention the following two. Choice of a verb head as a perspectivizing factor considerably reduces the possibility that the same polysemous object collocate is used in different senses with the same verb. Furthermore, the resulting clusters can give information about the senses, or meaning facets, of the verb head.

\subsection{Identification and ranking of noun clusters}

For the sake of concreteness, let us consider the following object-collocates of the Italian verb 


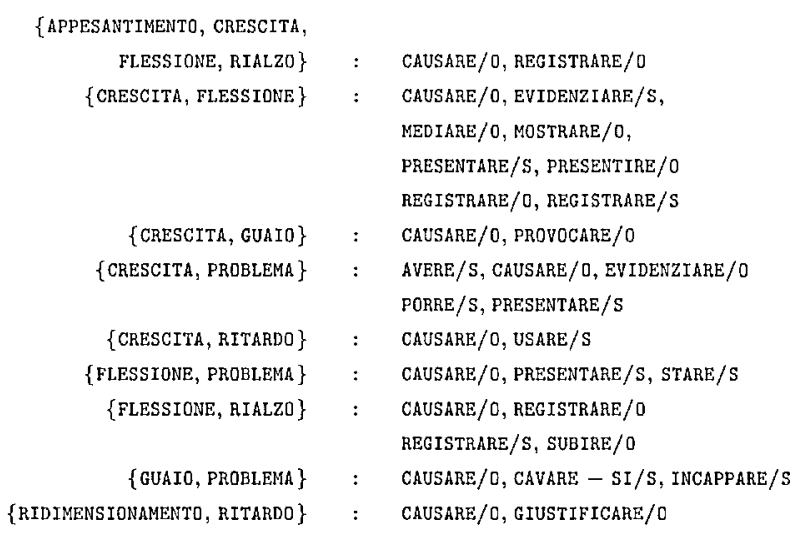

CAUSARE / 0 , REGISTRARE / 0

CAUSARE/0, EVIDENZIARE/S,

MEDIARE/0, MOSTRARE/ 0 ,

PRESENTARE/S, PRESENTIRE/ 0

REGISTRARE/O, REGISTRARE/S

CAUSARE / 0 , PROVOCARE/ 0

AVERE/S, CAUSARE / O, EVIDENZTARE /O

PORRE/S, PRESENTARE/S

CAUSARE / 0 , USARE/S

CAUSARE/O, PRESENTARE/S, STARE/S

CAUSARE/O, REGISTRARE/O

REGISTRARE/S, SUBIRE/O

CAUSARE/O, CAVARE - SI/S, INCAPPARE/S

CAUSARE/O, GIUST IFICARE/O

Figure 1: Some SIs relative to the collocates of the headword causare.

causare 'cause', as they are found in a test colpus:

appesantimento 'increase in weight', crescita 'growth', flessione 'decreasc', guaio 'trouble', problema 'problem', rialzo 'rise', ridimensionamento 'reduction', ritardo 'delay', turbolenza 'turbulence'.

Clustering these input words requires preliminary identification of Substitutability Islands $(S I \mathrm{~s})$. An example of $S I$ is the quadruple formed by the verb pair causare 'cause' and incappare 'run into' and the noun pair guaio 'troulble' and problema 'problem', where members of the same pair are inter-substitutable in context, given the constraints enforced by the $A P$ type in (2). Note that guaio and problema are objects of causare, and prepositional complements (headed by in 'in') of incappare. This makos it possible to maximize the similarity of trouble and problem across functionally heterogeneous contextis.

Bigger $S I$ s than the one just shown will form as many $A P$ s as there are quadruples of contextually interchangeable nouns and verbs. We consider a lexico-scmantic cluster of nouns the projection of an $S I$ onto the set of nouns. Fig.1 illustrates a sample of noun clusters (between curly brackets) projected from a set of $S I \mathrm{~s}$, together with a list of the verbs found in the same $S I$ s (the suffix ' $S$ ' stands for subject, and ' $O$ ' for object). Due to the asymmetry of classification, verbs in $S I$ s are not taken to form part of a lexico-semantic cluster in the same sense as nouns are.

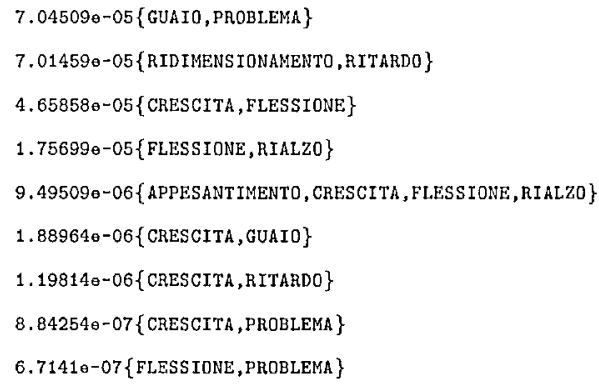

Figure 2: Nine top-most scored noun cluster's

Not all projected noun clusters exhibit the same degree of semantic coherence. Intuitively, the cluster appesantimento crescita flessione rialzo\} 'increase in weight, growth, decrease, rise' is semantically more appealing than the cluster $\{$ crescita problema\} 'growtih problem' (Fig.1).

A quantitative moasure of the semantic cohesion of a noun cluster $C_{N}$ is given by the correlation score $\sigma(S I)$ of the $S J$ of which $C_{N}$ is a projection. In Fig.2 noun clusters are ranked by decreasing values of $\sigma(S I)$, calculated according to eq. (9).

\subsection{Centroid identification}

Noun clusters of Figs. 1 and 2 are admittedly considerably fine grained. A coarser grain can be attained trivially through set union of intersecting clusters. In fact, what we want to obtain is a set of maximally orthogonal and semantically coherent noun classes, under the assumption that these classes highly corrclate with the principal meaning components of the verb head of which input nouns are objects.

In the algorithm evaluated here this is achieved in two steps: i) first, we select the best possible centroids of the prospective classes among the noun clusters of Fig.2; secondly, ii) we lump outstanding clusters (i.e. clusters which have not been selected in step i)) around the identified centroids. In what follows, we will only focus on step i). Results and evaluation of step ii) are reported in (Allegrini et al. 2000).

In step i) we assume that centroids are disjunctively defined, maximally coherent classes; hence, there exists no pair of intersecting centroids. The best possible selection of centroids will include non-intersecting clusters with the highest possible cumulative score. In practice, the best centroid corresponds to the 
cluster with the topmost $\sigma(S I)$. 'J'he second best centroid is the cluster with the second highest $\sigma(S I)$ and no intersection with the first centroid, and so on the $i$-th centroid is the $i$-th highest cluster with no intersection with the first $i-1$ centroids) until all chusters in the rank are used up. Clusters selected as centroids in the causare example above are: \{GUAIO PROBLEMA , \{RIDIMENSIONAMENTO RITARDO\}, \{CRESCITA FLESSIONE\}.

Clearly, this is not the only possible strategy for centroid selection, but certainly a suitable one given our assumptions and goals. To sum up, the targeted classification is local, i.e. conditional on a specific verb head, and orthogonal, i.e. it aims at identifying maximally disjunctive classes with high correlation with the principal meaning components of the verb head. 'This strategy leads to identification of the different: senses, or possibly moaning facets, of a verb. In their turn, noun clusters may capture subthe semantic distinctions. For instance, a distinction is made between incremental events or results of incremental events, which presuppose? a scalar dimension (as in the casse of ferescita flessione\} 'growth, decrease') and rescheduling cvents, where a change occurs with respect to a previously plamned event or object (see the centroid \{ridimensionamento ritardo\} 'reduction (clay').

\section{Experiment and evaluation}

We were able to extract all ST's relative to the entire $K B$. However, we report here an intrinsic: evaluation of the accuracy of acquired centroids which involves only a small subset of our results, since provision of a reference class typology is extremely labour-intensive. '

Wo consider 20 Italian verbs and their object collocates. ${ }^{2}$ The object collocates were automatically extracted from the "Italian SPARKLE Reference Corpus", a corpus of Italian financial

\footnotetext{
${ }^{1}$ For an extrinsic evaluation of the proposed similarity measure the reader is refered to (Montemagni et al. 1996; Briscoe et al. 1999; Federici et al. 1999a).

'The test verbs are: aggiungere 'add', aintare 'help', aspettare 'expect', cambiare 'change', causare 'canse', chicdere 'ask', considerare 'consider', dare 'give', decidere 'decide', fornire 'provide', muovere 'move', permeitcre 'allow', portare 'bring', produrre 'produce', sccglicre 'choose', sentire 'feel', stabilire 'establish', tagliare 'cut:', terminare 'end', trovare 'find'.
}

newspapers of about one million word tokens (Federici et al. 1998).

For each test verb, an independent classification of its collocates was created manually, by partitioning the collocates into disjoint sets of scmantically coherent lexical preferences, each set pointing to distinct senses of the test verb, according to a reference monolingual dictionary (Garyanti 1984). 'This considerably reduces the amount of subjectivity inevitably involved in the creation of a reference partition, and minimizes the probability that more than one sense of a polysemous noun can appear in the same class of collociates.

'The infered centroids, selected from clusters ranked by $\sigma(S I)$ defined as in (9), are projected against, the reference classification. Precision is defined as the ratio between the number of centroids properly included in one reforence class and the number of inferred centroids. Recall is defined as the ratio between the number of reference classes which properly include at least one centroid and the number of all reference classes.

Fig.3 shows results for the sets of object collocaties of polysemous test verbs only, as monosemous verbs trivially yickl $100 \%$ precision-recall. All average value over the sets of object collocates of all verbs is also shown, with $86 \%$ $88 \%$ of precision recall. Another average value is also plotted (as a black upright, triangle), obtained by ranking noun chusters by $\sigma(S I)$ calculated as in (10). This average value (53\%-53\% precision-recall) provides a sort of baseline of the difficultiy of the task, and sheds considerable light on the use of $A l^{\text {'s }}$, rather than simple verb-noun pairs, as information units for: measuring internal cohesion of centroids.

$$
\sigma(S I) \equiv \sum_{(n, v) \in S I} \sigma(n, v)
$$

\section{Conclusion}

We described a linguistic knowledge acquisition model and tested it on a word classification task. The main points of our proposal are:

- classification is asymmetric, grounded on principles of machine learning with infinite memory;

- the algorithm is explorative and nonreductionist; no a priori model of class dis- 


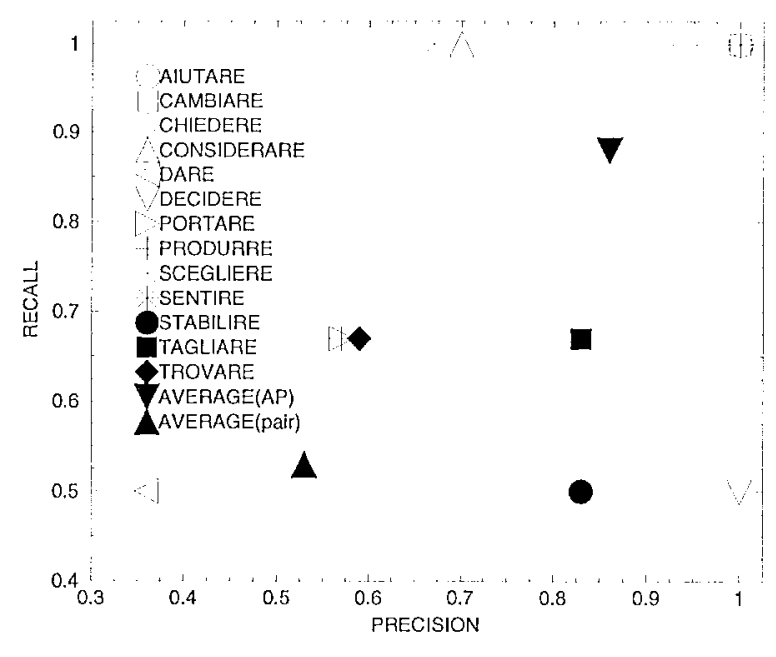

Figure 3: Centroid precision and recall for object collocates of polysemous verbs.

tribution is assumed;

- classification is modelled as the task of forming a web of context-dependent scmantic associations among words;

- the approach uses a context--sensitive notion of semantic similarity;

- the approach rests on the notion of analogical proportion, which proves to be a reliable information unit for measuring semantic similarity;

- analogical proportions are harder to track down than simple pairs, and interconnected in a highly complex way; yet, reliance, on data types, as opposed to token frequencies, makes the proposed method computationally tractable and resistant to data sparseness.

\section{References}

Allegrini P., Montemagni S., Pirrelli V. (2000) Controlled Bootstrapping of Lexico-semantic Classes as a Bridge between Paradigmatic and Syntagmatic Knowledge: Methodology and Evaluation. In Preceedings of LREC-2000, Athens, Greece May-June 2000, pp. 601-608.

Briscoe T., McCarthy D., Carroll J., Allegrini P., Calzolari N., Federici S., Montemagni S., Pirrelli V., Abney S., Beil F., Carroll G., Light, M., Prescher D., Riezler S., Rooth M. (1999) Acquisition System for Syntactic and Semantic Type and Selection. Deliverable 7.2. WP 7, EC project SPARKLE "Shallow Parsing and Knowledge Extraction for Language Engineering" (LE-2111).
Federici, S., Montemagni, S., Pirrelli, V. (1999) SENSE: an Analogy-based Word Sonse Disambiguation System. In M. Light and M. Palmer (eds.), Special Issuc of Natural Language Engineering on Lexical Scmantic Tagging.

Federici, S., Montemagni, S., Pirrelli, V., Calzolari, N. (1998) Analogy-based Extraction of Lexical Knowledge from Corpora: the SPARKLE Experience. In Proceedings of $L R E C-1998$, Granada, SP, May 1998.

Fodor, J.A. (1998) Concepts. Where Cognitive Science Went Wrong. Clarendon Press, Oxford, 1998.

Garzanti (1984) Il Nuovo Dizionario Italiano Garzanti. Garzanti, Milano, 1984.

Grefenstettc, G. (1994) Explorations in Automatic Thesaurus Discovery. Kluwer Academic Publishcrs, Boston, 1994.

Lin D. (1998) Automatic Retrieval and Clustering of Similar Words. In Proceedings of COLINGACL'98, Montreal, Canada, August 1998.

Miller, G.A., Charles, W.G. (1991) Contextual Correlates of Semantic Similarity. In Language and Cognitive Processes, 6 (1), pp. 1-28.

Montemagni, S. (1995) Subject and Object in Italian Sentence Processing. PhD Dissertation, UMIST, Manchester, UK, 1995.

Montemagni, S., Federici, S., Pirrelli, V. (1996) Resolving syntactic ambiguitics with lexicoscmantic pattcrns: an analogy-based approach. In Proceedings of COJING-96, Copenhagen, August 1996, pp. 376-381.

Pereira, F., Tishby, N. (1992) Distributional Similarity, Phase Transitions and Hierarchical Clustering. In Working Notes, Fall Symposium Series. AAAI, pp. 54-64.

Percira, F., Tishby, N., Lee, L. (1993) Distributional Clustering Of English Words. In Proceedings of the 30th Annual Meeting of the Association for Computational Linguistics, pp. 183-190.

Rooth, M. (Ms) Two-dimensional clusters in grammatical relations. In Symposium on Representation and Acquisition of Lexical Knowledge: Polyscmy, Ambiguity and Generativity, AAAI 1995 Spring Symposium Scries, Stanford University.

Rooth, M., Riczler, S., Prescher, D., Carroll, G., Beil, F. (1999) Inducing a Semanticallt Annotated Lexicon via EM-Based Clustering. In Proceedings of the 37th Annual Meeting of the Association for Computational Linguistics, Maryland, USA, Junc 1999, pp. 104-111.

Schütze, H., Pedersen, J. (1993) A vector model for syntagmatic and paradigmatic relatedness. In Proceedings of the 9th Annual Conference of the $U W$ Centre for the New OED and Text Rescarch, Oxford, England, 1993, pp. 104-113. 